Pacific Journal of Mathematics

DIVERGENCE OF COMPLEX RATIONAL APPROXIMATIONS 


\title{
DIVERGENCE OF COMPLEX RATIONAL APPROXIMATIONS
}

\author{
D. S. LUBINSKY
}

General rational interpolations, orthogonal-Padé approximations and best rational real approximations are shown to diverge as badly as classical Padé approximants. The examples also show known convergence results to be best possible in a strong sense.

1. Introduction. In [3], the author used extensions of Wallin's methods [10] to show that the well known Nuttall-Pommerenke theorem on convergence in capacity of Padé sequences is substantially best possible. One might expect that general rational interpolations with free poles, should fare better than classic Padé approximants, at least inside the closure of the interpolation points. Surprisingly they do not.

In this note, a new method is used to establish counterexamples to extension of known convergence results for (i) rational interpolants (ii) Padé-orthogonal approximations (iii) best rational real approximations. More specifically, it is shown that diagonal and non-diagonal rational sequences formed from entire functions may diverge in the limit on given $\sigma$-compact sets of capacity zero, and that diagonal sequences formed from functions with finite radius of analyticity may diverge in the limit on sets whose intersection with every open ball has positive area. Even in the classic Padé case, the latter example is more complete than Theorem 3 in [3]. It also settles conclusively a problem posed by Goncar ${ }^{1}$.

2. Notation. (i) Throughout $L, L_{i}, M, M_{i}, N, N_{i}$ denote positive integers and

$$
T(i)=L_{i}+M_{i}+1 \text {. }
$$

Further $I$ is a bounded real interval and for any function $f: I \rightarrow \mathbf{C}$, let $\|f\|=\sup \{|f(t)|: t \in I\}$. Also let $\|I\|=\sup \{|t|: t \in I\}$.

(ii) Given any integer $n \geq 1, \mathscr{P}_{n}$ is the class of polynomials of degree $n$ with 1 as (leading) coefficient of $z^{n}$. Also $\mathscr{P}_{0}=\{1\}$.

\footnotetext{
'A. A. Goncar, On the convergence of generalized Padé approximants to meromorphic functions, Math. USSR Sbornik, 27 (1975), 503-514. On page 504: "If $D(f)$ is a disc of finite radius..."
} 
(iii) For any Borel set $\mathcal{E} \subset \mathbf{C}, \operatorname{cap}(\mathcal{E})$ denotes the logarithmic capacity of $\mathcal{E}$.

(iv) A rational function $R=P / Q$ is of type $(L / M)$ if $\operatorname{deg}(P) \leq L$; $\operatorname{deg}(Q) \leq M ; Q \neq 0$. Further $R$ is real if $P, Q$ have real coefficients.

Definition 2.1. Let $\beta=\left\{\beta_{L M j}\right\}$ be complex number s.t. $\beta_{L M j}$ is given for each $1 \leq j \leq L+M+1$, all $L, M$. We assume for some $\Gamma \geq 1$

$$
\left|\beta_{L M J}\right| \leq \Gamma \quad \text { all } L, M, j
$$

and set

$$
\zeta(L / M)(z)=\prod_{j=1}^{L+M+1}\left(z-\beta_{L M_{J}}\right) \text { all } L, M .
$$

If $f: \beta \rightarrow \mathbf{C}$ is analytic at the zeroes of $\zeta(L / M)$ then $\mathscr{G}(L / M)=P / Q$ is the rational function of type $(L / M)$ s.t. $(f Q-P) / \zeta(L / M)$ is analytic at the zeroes of $\zeta(L / M)$.

See Wallin [9] for the convergence results for the rational interpolating functions $\mathscr{S}(L / M)$.

Definition 2.2. Let $\alpha: I \rightarrow R$ be non-decreasing with infinitely many points of increase. Let $\Phi_{J} \in \mathscr{P}_{J}\left(\right.$ all $j \geq 0$ ) satisfy $\int_{I} \Phi_{i} \Phi_{j} d \alpha=0$ all $i \neq j$.

Then given continuous $f: I \rightarrow \mathbf{C}$, the linear $\alpha$-Padé approximant $\langle[L / M]\rangle=P / Q$ and non-linear $\alpha$-Padé approximant $\langle\langle L / M\rangle\rangle$ are rational functions of type $(L / M)$ s.t.

$$
\begin{aligned}
\int_{I}(f Q-P) \Phi_{j} d \alpha=0 & \text { all } 0 \leq j \leq L+M, \\
\int_{I}(f-\langle\langle L / M\rangle\rangle) \Phi_{j} d \alpha=0 & \text { all } 0 \leq j \leq L+M .
\end{aligned}
$$

See [7] and Suetin [8] for convergence results for $\langle[L / M]\rangle,\langle\langle L / M\rangle\rangle$.

Definition 2.3. Let $f: I \rightarrow \mathbf{R}$ be continuous in $I$. The best rational real approximation $R(L / M)$ to $f$ is a real rational function of type $(L / M)$ s.t. $\|f-\Omega(L / M)\|=\min \|f-R\|$, the minimum being taken over all real rational functions $R$ of type $(L / M)$.

See [5] for some "overconvergence" results for $\Re(L / M)$. 
3. Construction of the functions. The examples are based on:

Lemma 3.1. Let $M, N>0$ and $L \geq N$. Let $C \neq 0$ and $Q \in \mathscr{P}_{N}$; $U \in \mathscr{P}_{L-N+1} ; W \in \mathscr{P}_{L+M+1}$. Let

$$
b=\max \{|z|:(U Q W)(z)=0\}
$$

and

$$
r>0 \text { and } s \geq \max \{1,2 b, 2 r\} .
$$

Then there exist polynomials $P^{*}$ of degree $\leq L$, and $Q^{*}$ of degree $M$ s.t.

$$
U Q^{*} Q=P^{*}+C W
$$

and

$$
\left|U Q^{*}\right|(z) \leq(3 s)^{L+M+1}|C| \quad \text { all }|z| \leq r .
$$

If $C, U, W, Q$ are real, so are $P^{*}, Q^{*}$.

Proof. Let $P^{*}$ be the polynomial of degree $\leq L$ that interpolates to $-C W$ at the $(L+1)$ zeroes of $U Q$. Then $Q^{*}=\left(P^{*}+C W\right) /(U Q)$ has degree $M$ and (3.3) follows. Further for $|z|<s$,

$$
\begin{aligned}
Q^{*}(z) & =(2 \pi i)^{-1} \int_{|t|=s}\left(P^{*}+C W\right)(t) /[(U Q)(t)(t-z)] d t \\
& =(2 \pi i)^{-1} \int_{|t|=s} C W(t) /[(U Q)(t)(t-z)] d t
\end{aligned}
$$

(as $P^{*}(t) /[(U Q)(t)(t-z)]$ is analytic in $t$ for $|t|>s$ and is $O\left(|t|^{-2}\right)$ as $|t| \rightarrow \infty)$. Then for $|z| \leq r,(3.1),(3.5)$ give

$$
\left|Q^{*} U\right|(z) \leq s|C|(s+b)^{L+M+1}(s-b)^{-(L+1)}(s-r)^{-1}(r+b)^{L-N+1}
$$

and (3.2) then gives (3.4).

Following is the construction for the interpolation examples:

Lemma 3.2. Let $\left\{L_{i}\right\},\left\{M_{i}\right\},\left\{N_{i}\right\}$ satisfy $M_{i} \geq N_{i}>0$ and

$$
L_{i}-N_{i}+1 \geq \sum_{j=1}^{i-1} T(j) \quad \text { all } i>1
$$


(where $T(j)$ is given by (2.1)). Let $Q_{i} \in \mathscr{P}_{N_{i}}$ have no zeroes in $\beta(i \geq 1)$. Let $\left\{s_{l}\right\}$ be a monotone increasing sequence s.t. $s_{l} \geq 2 \Gamma$ and

$$
s_{i} \geq 2 \max \left\{|z|: Q_{i}(z)=0\right\} \quad \text { all } i \geq 1
$$

and

$$
r=\lim _{i} s_{i} / 2 .
$$

Then there is a function $f$ analytic in $|z|<r$ s.t.

$$
f-g\left(L_{i} / M_{i}\right)=E_{i}+\left(6 s_{i}\right)^{-T(i)} \zeta\left(L_{\imath} / M_{\imath}\right) / Q_{i}
$$

where

$$
\lim _{i} \sup \left|E_{i}(z)\right|^{1 / T(l)} \leq 1 / 2 \quad \text { all }|z|<r
$$

the upper bound holding uniformly in compact subsets for large $i$.

Proof. Let $W_{i}=\zeta\left(L_{l} / M_{i}\right) ; U_{i}=z^{l(i)} \prod_{j=1}^{l-1} \zeta\left(L_{j} / M_{j}\right)$ all $i>1$, where $l(i)$ is chosen s.t. $\operatorname{deg}\left(U_{l}\right)=L_{i}-N_{i}+1$ (possible by (3.6)). Let

$$
C_{i}=\left(6 s_{i}\right)^{-T(i)} \quad \text { all } i \geq 1 .
$$

Then for each $i>1$, Lemma 3.1 with $Q=Q_{i}, U=U_{i}, W=W_{i}, C=C_{i}$ shows that there exists $P_{i}^{*}$ of degree at most $L_{i}, Q_{i}^{*}$ of degree $M_{l}$ s.t.

$$
U_{i} Q_{\imath}^{*} Q_{i}=P_{i}^{*}+C_{i} W_{i}
$$

and

$$
\left|U_{i} Q_{i}^{*}\right|(z) \leq 2^{-T(i)} \quad \text { all }|z| \leq s_{i} / 2
$$

(by (3.4), (3.11)). Let

$$
f(z)=\sum_{j=2}^{\infty} U_{j} Q_{j}^{*}
$$

and for each $i>1$, let

$$
E_{i}=\sum_{j=i+1}^{\infty} U_{j} Q_{j}^{*}
$$

and

$$
P_{i}=Q_{i} \sum_{j=2}^{i-1} U_{j} Q_{j}^{*}+P_{i}^{*}
$$


Now we see that (3.6) implies $T(i)>i$, so (3.10) follows from (3.13) and it also follows that $f$ is analytic in $|z|<r$. Using (3.12), (3.14), (3.15), (3.16), we see

$$
f Q_{i}-P_{i}=E_{i} Q_{i}+C_{i} W_{i}
$$

It is easily seen from the definition of $U_{j}, W_{i}$ that the right member of (3.17) has zeroes when $\zeta(L / M)$ does; and (3.6) shows that $\operatorname{deg}\left(P_{i}\right) \leq L_{i}$, while $\operatorname{deg}\left(Q_{i}\right) \leq M_{i}$ so $\zeta\left(L_{i} / M_{i}\right)=P_{i} / Q_{i}$ by uniqueness. Finally (3.11), (3.17) $\Rightarrow(3.9)$.

Note that in the Newton-Padé case (all $\beta_{L M j}=\beta_{j}$ ) we could just take $L_{i}-N_{i}+1 \geq T(i-1)$ and $U_{i}=z^{l(i)} \zeta\left(L_{i} / M_{i}\right)$. Following is the construction for the Padé-orthogonal examples:

LEMMA 3.3. Let $\left\{L_{l}\right\},\left\{M_{i}\right\},\left\{N_{i}\right\}$ satisfy $M_{i} \geq N_{i}$ and

$$
L_{i}-M_{i}-N_{i} \geq L_{i-1}+M_{i-1}+N_{t-1} \text { all } i \geq 1 .
$$

Let $Q_{i} \in \mathscr{P}_{N_{t}}$ be real all $i \geq 1$. Let $\left\{s_{l}\right\}$ be monotone increasing and satisfy $s_{i} \geq 2\|I\|$ and (3.7). Let $r$ be given by (3.8).

(a) There is a function $f$ analytic in $|z|<r$ and real in $(-r, r)$ s.t.

$$
f-\left\langle\left[L_{i} / M_{i}\right]\right\rangle=E_{i}+\left(6 s_{i}\right)^{-T(i)} \Phi_{T(i)} / Q_{i}
$$

all $|z|<r, i>1$, where (3.10) holds.

(b) If the $\left\{Q_{i}\right\}$ have no zeroes in $I$, then there is a function $f$ analytic in $|z|<r$ and real in $(-r, r)$ s.t.

$$
f-\left\langle\left\langle L_{i} / M_{i}\right\rangle\right\rangle=E_{i}+\left(6 s_{i}\right)^{-T(i)} W_{i} / Q_{i}
$$

all $|z|<r, i>1$, where (3.10) holds and $W_{i} \in \mathscr{P}_{T(i)}$ has all its zeroes in $I$.

Proof. (a) Let $U_{l}=\Phi_{L_{i}-N_{i}+1}, W_{i}=\Phi_{T(l)}$ and $C_{i}$ be given by (3.11) all $i \geq 1$. Lemma 3.1 with $U=U_{i}, W=W_{i}, Q=Q_{i}, C=C_{i}$, show that there exist $P_{i}^{*}, Q_{i}^{*}$ s.t. both (3.12), (3.13) hold. Let $f, E_{i}, P_{i}$ be given by (3.14), (3.15), (3.16) respectively. We see that (3.10), (3.17) hold and so

$$
\begin{aligned}
\int_{I}\left(f Q_{i}-P_{i}\right) \Phi_{k} d \alpha & =\sum_{j=i+1}^{\infty} \int_{I} U_{j} Q_{j}^{*} Q_{i} \Phi_{k} d \alpha+C_{i} \int_{I} \Phi_{T(i)} \Phi_{k} d \alpha \\
& =0 \quad \text { for } 0 \leq k<T(i)
\end{aligned}
$$

(by (3.18) and choice of $U_{j}$ ). 
(b) Let $U_{i}, C_{i}$ be as in (a) but define $W_{i}$ differently: Since $d \alpha(x) / Q_{i}(x)$ is of one sign in $I$ with infinitely many points of change, there exists $W_{i} \in \mathcal{P}_{T(i)}$ s.t. $\int_{I} W_{i} P d \alpha / Q_{i}=0$ for all polynomials $P$ s.t. $\operatorname{deg}(P)<T(i)$. Then all $W_{i}$ 's zeroes lie in $I$. Further with $f, E_{i}, P_{l}$ given by (3.14), (3.15), (3.16) respectively we see

$$
\begin{aligned}
\int_{I}\left(f-P_{i} / Q_{i}\right) \Phi_{k} d \alpha & =\sum_{j=i+1}^{\infty} \int_{I} U_{j} Q_{j}^{*} \Phi_{k} d \alpha+C_{i} \int_{I} W_{i} \Phi_{k} d \alpha / Q_{i} \\
& =0 \quad \text { all } 0 \leq k<T(i) .
\end{aligned}
$$

Following is the construction for the best approximation examples.

LEMMA 3.4. Let $\left\{L_{i}\right\},\left\{M_{i}\right\},\left\{N_{i}\right\}$ satisfy $M_{i} \geq N_{i}$ and

$$
L_{i}-N_{i}-1 \geq 2 \sum_{j=1}^{i-1}\left(L_{j}+M_{j}+N_{j}\right) \quad \text { all } i>1 .
$$

Let $Q_{i} \in \mathscr{P}_{N_{i}}$ be real with no zeroes in $I(i \geq 1)$. Let monotone increasing $\left\{s_{i}\right\}$ satisfy

$$
s_{i} \geq 24 \max \left\{1,\|I\|, \max \left\{|z|: Q_{i}(z)=0\right\}\right\}
$$

and let $r$ be given by (3.8).

Then there is a function $f$ analytic in $|z|<r$ and real in $(-r, r)$ s.t.

$$
f-\Re\left(L_{i} / M_{i}\right)=E_{i}+\left(3 s_{i}\right)^{-2 T(i)} W_{i} / Q_{i}
$$

in $|z|<r$ where (3.10) holds and $W_{i} \in \mathscr{P}_{T(i)}$ has all its zeroes in $I(i \geq 1)$.

Proof. Let $C_{i}=\left(3 s_{i}\right)^{-2 T(i)}$ all $i \geq 1$. Let $W_{i}(z)=z^{T(i)}+P_{i}^{\#}(z)$ where $\operatorname{deg}\left(P_{i}^{\#}\right) \leq T(i)-1$ and $\left\|W_{i} / Q_{l}\right\|=\min \left\|\left(z^{T(i)}+P\right) / Q_{i}\right\|$ the minimum being taken over all polynomials $P$ s.t. $\operatorname{deg}(P) \leq T(i)-1$. Then Achieser $\left[1\right.$, p. 55] shows that $W_{i} / Q_{i}$ equioscillates at $T(i)+1$ points in $I$. Further then all $W_{i}$ 's zeroes lie in $I$. Thus (3.22) and $T(i)>2 N_{i}$ and Theorem 4 in Walsh [11, p. 104] show that all zeroes of the derivative $\left(W_{i} / Q_{i}\right)^{\prime}$ lie in the region $\left\{z:|z| \leq s_{i} / 8\right\}$. So

$$
\left(W_{i} / Q_{i}\right)^{\prime}=\left(T(i)-N_{i}\right) H_{i} / Q_{i}^{2}
$$

where $H_{i} \in \mathscr{P}_{L_{i}+M_{i}+N_{i}}$ is real and has at least $T(i)+1$ zeroes at the points of equioscillation of $W_{l} / Q_{i}$ in $I$, the remaining at most $N_{i}-2$ zeroes lying in $|z| \leq s_{i} / 8$. Then $|z|=s_{i} / 4$ implies

$$
\left|\left(W_{i} / Q_{i}\right)^{\prime}\right|(z)>\left(5 s_{i} / 24\right)^{T(i)+1}\left(s_{i} / 8\right)^{N_{i}-2}\left(7 s_{l} / 24\right)^{2 N_{t}}>1
$$


(as $s_{i} \geq 24$ and $T(i)+1 \geq 2 N_{i}+2$ ). Next let

$$
U_{i}(z)=z^{l(i)}\left(\prod_{j=1}^{i-1} H_{j}^{2}(z)\right)(z-\inf I)(z-\sup I)
$$

where $l(i)$ is determined so that $\operatorname{deg}\left(U_{i}\right)=L_{i}-N_{i}+1$ (possible by (3.21)). Then for each $i \geq 1$, Lemma 3.1 with $Q=Q_{i}, U=U_{i}, W=W_{i}$, $C=C_{i}$ gives polynomials $P_{i}^{*}, Q_{i}^{*}$ s.t. (3.12) holds and s.t.

$$
\max \left\{\left|U_{i} Q_{i}^{*}\right|(z):|z| \leq s_{i} / 2\right\} \leq\left(3 s_{i}\right)^{-T(i)} \quad \text { all } i \geq 1
$$

Let $f, E_{i}, P_{i}$ be given by (3.14), (3.15), (3.16) respectively. It remains to show $P_{i} / Q_{i}=\mathcal{R}\left(L_{i} / M_{i}\right)$. Cauchy's integral formula for $E_{i}^{\prime}$ applied to the contour $\left\{t:|t|=s_{i} / 2\right\}$ gives for $|z| \leq s_{i} / 4$,

$$
\begin{aligned}
\left|E_{i}^{\prime}(z)\right| & \leq\left(s_{i} / 2\right) \sum_{j=i+1}^{\infty} \max \left\{\left|U_{j} Q_{j}^{*}\right|(t):|t|=s_{i} / 2\right\}\left(s_{i} / 4\right)^{-2} \\
& \leq(1 / 3) \sum_{j=i+1}^{\infty}\left(3 s_{j}\right)^{-T(j)}
\end{aligned}
$$

(by monotonicity of $\left\{s_{j}\right\}$ and by (3.27)) $<C_{i} / 3 \quad$ (by choice of $C_{i}$ and as $T(j)>2 T(j-1)$ )

So for $|z|=s_{i} / 4,\left|E_{i}^{\prime}(z)\right|<\left|\left(C_{i} W_{i} / Q_{i}\right)^{\prime}(z)\right|$ (by (3.25)). Then Rouche's Theorem and analyticity of $E_{i}$ shows that $\left(E_{i}+C_{i} W_{i} / Q_{i}\right)^{\prime}$ has the same number of zeroes as $\left(C_{i} W_{i} / Q_{i}\right)^{\prime}$ in $\left\{z:|z|<s_{i} / 4\right\}$. But $E_{i}$ and $E_{i}^{\prime}$ vanish at all zeroes of $\left(C_{i} W_{i} / Q_{i}\right)^{\prime}$ (by (3.15), (3.24), (3.26)) while $E_{i}$ vanishes at $I$ 's endpoints. We deduce that $f-P_{i} / Q_{i}=E_{i}+C_{t} W_{i} / Q_{i}$ equioscillates at the at least $T(i)+1$ points of equioscillation of $C_{i} W_{i} / Q_{i}$ in $I$. Hence $P_{i} / Q_{i}=\Re\left(L_{i} / M_{i}\right)$.

Finally, we define some polynomials.

LEMMA 3.5. Let $\left\{L_{i}\right\},\left\{M_{i}\right\},\left\{N_{i}\right\}$ satisfy for some $c>0, \eta>0$

$$
\begin{gathered}
N_{i} \geq c T(i), \\
L_{i} \geq(1+\eta) T(i-1) .
\end{gathered}
$$

Let $a>1$ and $0<\varepsilon<1<r$. Let $\mathfrak{V}$ be a subset of $\{z:|z|<r\}$ with empty interior and $\mathcal{Q}=\{z:|z|<r\} \backslash \overline{\mathcal{V}}$ be non-empty. Let $W_{i} \in \mathscr{P}_{T(i)}$ have all its zeroes in $\overline{\mathcal{V}}(i \geq 1)$. 
Then there exist real polynomials $Q_{i} \in \mathscr{P}_{N_{i}}$ with zeroes in $\{z:|z|<r\} \backslash \mathscr{V}$ s.t. if

$$
\mathcal{E}_{i}=\left\{z:\left|W_{i} / Q_{i}\right|(z)>a^{T(i)}\right\} \quad \text { all } i \geq 1
$$

then

(a) If $B$ is a ball s.t. $B \cap$ Q is non-empty, then there is a ball $B^{\prime}$ that is non-empty s.t. $B^{\prime} \subset \mathcal{E}_{i} \cap B$ for all large $i$.

(b) If $B$ is a ball in $\{z:|z|<r\}$ s.t. $d(B) \geq \varepsilon$ then

$$
\lim _{i} \inf \operatorname{meas}\left(\mathcal{E}_{i} \cap B\right)>0
$$

where meas denotes planar Lebesgue measure.

Proof. We set $Q_{i}=Q_{1 i} Q_{2 i}$ where $Q_{1 i}$ is used to give (a) and $Q_{2 i}$ is used to give (b). Construction of $\left\{Q_{1 i}\right\}$.

Choose $\left\{x_{i}\right\}$ dense in, and contained in $\mathcal{Q}$. Set $\rho(i)=(\eta / 4)(1+\eta)^{-i}$ all $i$ so

$$
\sum_{i=1}^{\infty} \rho(i)=1 / 4
$$

Choose positive $\left\{r_{i}\right\}$ s.t. for all $i$

(3.32) $\left|x_{i}\right|+r_{i}<r$ and $r_{i}^{\rho(i) / 2}<\left(d\left(x_{i}, \overline{\mathcal{V}}\right) /(2 a)\right)^{1 / c}(2 r)^{-2}$

where $d\left(x_{i}, \overline{\mathcal{V}}\right)$ denotes the distance from $x_{i}$ to $\overline{\mathcal{V}}$. Let $n(i ; j)=$ greatest integer $\leq \rho(j) N_{i}$ all $1 \leq j \leq i$. Using (3.28), (3.29) and (3.31) we see

$$
n(i ; j) \geq \rho(j) N_{i} / 2 \text { all } 1 \leq j \leq i / 2
$$

(for large $i$ ). Now let

$$
Q_{1 i}(z)=(z-r)^{m(i)} \prod_{j=1}^{i}\left(\left(z-x_{j}\right)\left(z-\bar{x}_{j}\right)\right)^{n(i ; j)}
$$

where $m(i)$ is chosen s.t. $\operatorname{deg}\left(Q_{1 i}\right)=$ greatest integer $\leq N_{i} / 2$, this being possible by (3.31). Then $Q_{1 i}$ is a real polynomial with zeroes in $Q$. Let $B_{i}$ be the open ball of radius $r_{i}$, center $x_{i}$, all $i \geq 1$. Then $1 \leq j \leq i / 2$ and $z \in B_{j}$ implies

$$
\begin{aligned}
\left|Q_{1 i(z)}\right| & \leq r_{j}^{n(i ; j)}(2 r)^{N_{i} / 2} \\
& <\left(d\left(x_{j}, \overline{\mathcal{V}}\right) /(2 a)\right)^{N_{i} / c}(2 r)^{-N_{t}} \\
& <\left|W_{i}(z)\right| a^{-T(i)}(2 r)^{-N_{l}}
\end{aligned}
$$


(by (3.28) and as if $u$ is a zero of $W$ then $u \in \overline{\mathcal{V}}$ so $|z-u| \geq\left|u-x_{j}\right|-$ $\left.\left|x_{j}-z\right| \geq d\left(x_{j}, \overline{\mathcal{V}}\right)-r_{j} \geq d\left(x_{j}, \overline{\mathcal{V}}\right) / 2\right)$. Then as $\operatorname{deg}\left(Q_{2 i}\right)<N_{i}$ and its zeroes will lie in $|z|<r$, we deduce from (3.30) that $\cup_{j=1}^{i / 2} B_{j} \subset \mathcal{E}_{i}$ for all large enough $i$. As the $\left\{x_{i}\right\}$ are dense in $\mathcal{Q}$ and $r_{t} \rightarrow 0$, (a) follows.

Construction of $\left\{Q_{2_{l}}\right\}$. Choose $0<\varepsilon_{0}<\varepsilon / 30$ s.t.

$$
n\left(10 \varepsilon_{0}\right)=2 r
$$

for some integer $n$ and choose $\delta>0$ so small that $\delta / \varepsilon_{0}$ is a positive integer $>1$ and

$$
\varepsilon_{0}^{1 / c} \delta^{-1 /\left(8 n^{2}\right)}(4 r)^{-1}>a^{1 / c}(2 r) .
$$

Let $\delta=\{z:|\operatorname{Re}(z)| \leq r ;|\operatorname{Im}(z)| \leq r\}$ and divide $\delta$ into $n^{2}$ squares $S(1) \cdots S\left(n^{2}\right)$ of side $10 \varepsilon_{0}$. Let

$$
\mathcal{L}_{i}=\left\{z:\left|W_{i}(z)\right| \leq \varepsilon_{0}^{T(t)}\right\} \quad \text { all } i \geq 1
$$

which implies meas $\left(\mathcal{L}_{l}\right) \leq 4 e \pi \varepsilon_{0}^{2}$ all $i \geq 1$ (by Cartan's Lemma [2, p. 194]). Next, for $i \geq 1,1 \leq j \leq n^{2}$, meas $(S(j))=100 \varepsilon_{0}^{2}>2 \operatorname{meas}\left(\varrho_{i}\right)$ so we can find a square $S(i ; j)$ of centre $b(i ; j)$ side $\delta$ s.t.

$$
\begin{aligned}
& S(i ; j) \subset S(j) \text { and } \operatorname{meas}\left(S(i ; j) \backslash \mathcal{L}_{i}\right)>\delta^{2} / 2 \\
&\left(1 \leq j \leq n^{2} ; i \geq 1\right) .
\end{aligned}
$$

By displacing the $b(i ; j)$ slightly we can assume all $b(i ; j) \notin \mathfrak{V}$. Let

$$
Q_{2 i}(z)=(z-r)^{q(i)} \prod_{j=1}^{n^{2}}\{(z-b(i ; j))(z-\bar{b}(i ; j))\}^{p(t)}
$$

where $p(i)=$ greatest integer $\leq N_{i} /\left(4 n^{2}\right)$ and $q(i)$ is determined so that $\operatorname{deg}\left(Q_{1}, Q_{2 i}\right)=N_{l}$. Then $z \in \cup_{j=1}^{n^{2}} S(i ; j) / \ell_{\iota}$ implies

$$
\left|W_{\iota} / Q_{2 i}\right|(z)>\varepsilon_{0}^{T(i)} /\left\{\delta^{p(\imath)}(4 r)^{N_{\imath}}\right\}>a^{T(\imath)}(2 r)^{N_{\iota}} .
$$

(as $p(i) \geq N_{t} /\left(8 n^{2}\right)$ and by (3.28), (3.35)). As before this implies $z \in \mathcal{E}_{l}$. Finally if $B \subset\{z:|z|<r\}$ and $d(B) \geq \varepsilon$, then (3.34) implies that $B$ must contain some $S(j)$. Then for all $i$, meas $\left(B \cap \mathcal{E}_{i}\right) \geq \operatorname{meas}\left(S(j) \cap \mathcal{E}_{l}\right) \geq$ $\operatorname{meas}\left(S(i ; j) \backslash \mathcal{E}_{l}\right)>\delta^{2} / 2($ by $(3.37))$.

4. Results. We show now that for functions $f$ analytic in a large circle centre 0 (but with singularities of positive capacity in C) diagonal sequences of rational approximations will not in general converge in measure or in capacity in any open set within $f$ 's radius of analyticity. So 
the requirement of singularities of cap 0 in $\mathbf{C}$ in the various Nuttall-Pommerenke theorems $[5,7,9]$ is essential. Even for the usual Padé approximants (where all $\beta_{L M j}=0$ ) this provides a more complete counterexample than Theorem 3 in [3], which did not exclude the possibility that the Padé approximants converged in measure in a neighbourhood of zero.

THEOREM 4.1. Let $0<\varepsilon<1<r$. Let $\left\{L_{k}\right\},\left\{M_{k}\right\}$ satisfy for some $\lambda \geq 1, \eta>1$

$$
\begin{array}{rlrl}
1 / \lambda & \leq M_{k} / L_{k} \leq \lambda & & \text { all } k \geq 1, \\
L_{k} \geq(1+\eta) T(k-1) & & \text { all } k>1 .
\end{array}
$$

Then there is a function $f$ analytic in $|z|<r$ s.t. if $R_{k}=9\left(L_{k} / M_{k}\right)$ all $k \geq 1$, and if

$$
\mathscr{F}_{k}=\left\{z:|z|<r \text { and }\left|f-R_{k}\right|(z)>2^{T(k)}\right\} \quad \text { all } k \geq 1
$$

then

(a) for any ball $B$ s.t. $B \cap\{z:|z|<r\} \backslash \bar{\beta} \neq \varnothing$ there exists a ball $B^{\prime} \neq \varnothing$ s.t. $B^{\prime} \subset \mathscr{F}_{k} \cap B$ for large $k$.

(b) for any ball $B$ in $\{z:|z|<r\}$ s.t. $d(B) \geq \varepsilon$, we have

$$
\lim _{k} \inf \operatorname{meas}\left(\mathscr{F}_{k} \cap B\right)>0 .
$$

Hence $\left\{R_{k}\right\}$ (and all its subsequences) cannot converge in measure in any open set in $\{z:|z|<r\} \backslash \bar{\beta}$ nor in any ball $B$ in $\{z:|z|<r\}$ s.t. $d(B) \geq \varepsilon$.

Proof. Let

$$
N_{k}=\text { greatest integer } \leq \min \left\{M_{k},(1-1 / \eta) L_{k}\right\} \quad \text { all } k \geq 1 .
$$

Then (4.2) implies $T(j)<(1+\eta)^{-1} T(j+1)$, so

$$
\sum_{j=1}^{i-1} T(j)<T(i-1)(1+\eta) / \eta \leq L_{i} / \eta<L_{i}-N_{i}+1
$$

(by (4.4)). Hence (3.6) holds. Let $W_{i}=\zeta\left(L_{i} / M_{i}\right)$ all $i \geq 1$ and $a=25 r$ and $\mathcal{V}=\beta$ in Lemma 3.5. Since (4.1), (4.2), and (4.4) hold, Lemma 3.5 gives real polynomials $Q_{i} \in \mathscr{P}_{N_{i}}$ satisfying Lemma 3.5(a), (b) and with all their zeroes in $\{z:|z|<r\} \backslash \beta$. We can clearly assume $r>\Gamma$ (given by (2.2)). Letting $s_{i}=2 r$ all $i$, we see (3.7) holds. Then with the $\left\{L_{i}\right\},\left\{M_{i}\right\}$, $\left\{N_{i}\right\},\left\{W_{i}\right\},\left\{Q_{i}\right\}$ chosen above, Lemma 3.2 gives a function $f$ analytic in 
$|z|<r$ s.t. (3.9), (3.10) hold. Then for all $|z| \leq r^{\prime}<r$ s.t. $z \in \mathcal{E}_{i}$ (given by (3.30)) we have for large $i$

$$
\left|f-\mathscr{G}\left(L_{i} / M_{i}\right)\right|(z) \geq(12 r)^{-T(i)}\left|\zeta\left(L_{i} / M_{i}\right) / Q_{i}\right|(z)-1>2^{T(i)}
$$

(by (3.30) and as $a=25 r$ ). Thus $\mathcal{E}_{i} \cap\left\{z:|z| \leq r^{\prime}\right\} \subset \mathscr{F}_{i}$ for large $i$ and Lemma 3.5(a), (b) give our result.

REMARKS. (a) So surprisingly enough, there is not even convergence in measure in $\bar{\beta}$, the closure of the interpolation points, in general.

(b) One may modify the above example so that divergence of the above type occurs even when most of the poles of the $\left\{9\left(L_{k} / M_{k}\right)\right\}$ are fixed in advance and are not determined by interpolatory conditions-see the technical report [6] (not intended for publication).

THEOREM 4.2. Let $r>1$. There is a function $f$ analytic in $|z|<r$ and real in $(-r, r)$ s.t. if $\left\{R_{k}\right\}=\left\{\left\langle\left[L_{k} / M_{k}\right]\right\rangle\right\}$ (or $\left\{R_{k}\right\}=\left\{\left\langle\left\langle L_{k} / M_{k}\right\rangle\right\rangle\right\}$; or $\left.\left\{R_{k}\right\}=\left\{\Re\left(L_{k} / M_{k}\right)\right\}\right)$ then for any ball $B$ s.t. $B \cap\{z:|z|<r\} \neq \varnothing$ there exists a ball $B^{\prime} \neq \varnothing$ s.t. $B^{\prime} \subset \mathscr{F}_{k} \cap B$ for large $k$ (where $\mathscr{F}_{k}$ is given by (4.3)).

Hence $\left\{R_{k}\right\}$ (and all its subsequences) cannot converge in measure or in capacity to $f$ in any subset of $\{z:|z|<r\}$ with non-empty interior.

Here (a) If $\left\{R_{k}\right\}=\left\{\left\langle\left[L_{k} / M_{k}\right]\right\rangle\right\}$ or $\left\{R_{k}\right\}=\left\{\left\langle\left\langle L_{k} / M_{k}\right\rangle\right\rangle\right\}$ we must insist that for some $\eta>0, \lambda \geq 1$,

$$
M_{k} / \lambda \leq L_{k}-M_{k} \leq \lambda M_{k} ; L_{k}-M_{k} \geq(1+\eta) T(k-1) .
$$

(b) If $\left\{R_{k}\right\}=\left\{\Re\left(L_{k} / M_{k}\right)\right\}$ we must insist that (4.1), (4.2) hold for some $\eta>2, \lambda \geq 1$.

Proof. Similar to Theorem 4.1, but using Lemmas 3.3, 3.4.

REMARKs. (a) While (4.5) excludes the case $L_{i}=M_{i}$, it allows $L_{i}=$ $(1+\delta) M_{i}$ where $\delta>0$ may be arbitrarily small.

(b) For the linear Padé approximants $\left\{\left\langle\left[L_{k} / M_{k}\right]\right\rangle\right\}$ the example can be modified to show that there is not even convergence in capacity in any segment of $I$ with diameter $\geq \varepsilon$ ( $\varepsilon$ being fixed in advance).

(c) We next show that general diagonal rational sequences formed from entire functions can diverge in the limit on given $\sigma$-compact sets of cap 0. As in [3] (where this was shown for the usual Padé approximants) this implies that convergence in cap cannot be strengthened to convergence in some "thinner" set function. 
THEOREM 4.3. Let $\mathcal{E}$ be $\sigma$-compact and $\operatorname{cap}(\mathcal{E})=0$. Then there is an entire function $f$ s.t.

$$
\lim _{k}\left|f-R_{k}\right|(z)^{1 / T(k)}=\infty \quad \text { all } z \in \mathcal{E} .
$$

Here (a) In the interpolation case, we assume $\mathcal{E} \subset \mathbf{C} \backslash \bar{\beta}$ and $\left\{R_{k}\right\}=$ $\left\{9\left(L_{k} / M_{k}\right)\right\}$ where (4.1), (4.2) hold for some $\lambda>1, \eta>1$.

(b) In the orthogonal-Pade approximation case, we assume $\mathcal{E} \subset \mathbf{C} \backslash I$ and $\left\{R_{k}\right\}=\left\{\left\langle\left[L_{k} / M_{k}\right]\right\rangle\right\}$ (or $\left.\left\{R_{k}\right\}=\left\{\left\langle\left\langle L_{k} / M_{k}\right\rangle\right\rangle\right\}\right)$ where (4.5) holds for some $\lambda \geq 1, \eta>0$.

(c) In the best approximation case, we assume $\mathcal{E} \subset \mathbf{C} \backslash I$ and $\left\{R_{k}\right\}=$ $\left\{\Re\left(L_{k} / M_{k}\right)\right\}$ where (4.1), (4.2) hold for some $\lambda \geq 1, \eta>2$.

Proof. (a) With $\left\{N_{k}\right\}$ given by (4.4), we see as before that (3.6) holds. Further there exists $c>0$ s.t. (3.28) holds. Next the arguments in [3, Theorem 2] show that there exist $Q_{k} \in \mathscr{P}_{N_{k}}($ all $k \geq 1)$ and $\left\{\varepsilon_{k}\right\}$ s.t. $\lim _{k} \varepsilon_{k}=0$, s.t.

$$
\begin{gathered}
Q_{k} \text { 's roots lie in }|z| \leq \varepsilon_{k}^{-c / 3} / 2 \text { all large enough } k, \\
z \in \mathcal{E} \text { implies }\left|Q_{k}(z)\right|<\varepsilon_{k}^{N_{k}} \text { all large enough } k .
\end{gathered}
$$

By minute displacements to the zeroes of $\left\{Q_{k}\right\}$, we may assume that they lie outside $\beta$. Set $s_{k}=\varepsilon_{k}^{-c / 3}$ all $k \geq 1$ in Lemma 3.2. Then (2.2) and (4.7) imply (3.7). Further $\lim _{k} s_{k}=\infty$, so $f$ of Lemma 3.2 is entire. Further $z \in \mathcal{E}$ and (3.9), (3.10), (3.28), (4.8) give for large $k$,

$$
\begin{gathered}
\left|f-g\left(L_{k} / M_{k}\right)\right|(z)^{1 / T(k)} \geq\left\{\left(6 s_{k}\right)^{-T(k)}\left|\zeta\left(L_{k} / M_{k}\right) / Q_{k}\right|(z)-1\right\}^{1 / T(k)} \\
\geq 0.1 \varepsilon_{k}^{c / 3-c}\left|\zeta\left(L_{k} / M_{k}\right)\right|(z)^{1 / T(k)} \rightarrow \infty \quad \text { as } k \rightarrow \infty
\end{gathered}
$$

as $\zeta\left(L_{k} / M_{k}\right)$ 's zeroes lie in $\beta$ and $z \notin \bar{\beta}$. (b), (c) are similar, though one replaces $Q_{k}$ above by $Q_{k}=Q_{k}^{*}(z) \bar{Q}_{k}^{*}(\bar{z})$ where $Q_{k}^{*} \in \mathscr{P}_{N_{k} / 2}$ to obtain real $Q_{k}$.

Remarks. (a) One can relax $\varepsilon \subset \mathbf{C} \backslash \bar{\beta}$ slightly and also allow the zeroes of $\zeta\left(L_{k} / M_{k}\right)$ to tend to $\infty$ sufficiently slowly (the rate depending on $\mathcal{E}$ ). Similarly for the linear Padé approximants, one can relax $\mathcal{E} \subset \mathbf{C} \backslash I$.

(b) It is well known that non-diagonal sequences of various rational approximations converge in capacity-in fact, this is best possible:

THEOREM 4.4. Let $\mathcal{E}$ be a $\sigma$-compact set s.t. $\operatorname{cap}(\mathcal{E})=0$. There exists an entire function $f$ and $\left\{M_{k}\right\},\left\{L_{k}\right\}$ s.t. $\lim _{k} L_{k}=\infty ; \lim _{k} M_{k} / L_{k}=0$ and $\lim _{k}\left|f-R_{k}\right|(z)^{1 / T(k)}=\infty$ all $z \in \mathcal{E}$. 
Here (a) In the interpolation case, we assume $\mathcal{E} \subset \mathbf{C} \backslash \bar{\beta}$ and $\left\{R_{k}\right\}=$ $\left\{9\left(L_{k} / M_{k}\right)\right\}$.

(b) In the orthogonal-Pade approximation case, we assume $\mathcal{E} \subset \mathbf{C} \backslash I$ and $\left\{R_{k}\right\}=\left\{\left\langle\left[L_{k} / M_{k}\right]\right\rangle\right\}\left(\right.$ or $\left.\left\{R_{k}\right\}=\left\{\left\langle\left\langle L_{k} / M_{k}\right\rangle\right\rangle\right\}\right)$.

(c) In the best approximation case, we assume $\mathcal{E} \subset \mathbf{C} \backslash I$ and $\left\{R_{k}\right\}=$ $\left\{\Re\left(L_{k} / M_{k}\right)\right\}$.

Proof. This is similar that of Theorem 4.3-a full proof appears in [6].

Note finally that the results in [4] which characterize the thinness of exceptional sets for non-diagonal sequences of Padé approximants also hold for the above approximations-see [6].

5. Acknowledgement. I would like to thank Professor Oved Shisha for referring me to [11].

\section{REFERENCES}

[1] I. N. Achieser, Theory of Approximation, Ungar, New York, 1956.

[2] G. A. Baker, Jr., Essentials of Padé Approximants, Academic Press, New York, 1975.

[3] D. S. Lubinsky, Diagonal Padé approximants and capacity, J. Math. Anal. Appl., 78 (1980), 58-67.

[4] On non-diagonal Padé approximants, J. Math. Anal. Appl., 78 (1980), 405-428.

[5] On convergence of rational and best rational approximations, Technion Mathematics Preprint MT-508(1981).

[6] Counterexamples in complex rational approximation, Technion Mathematics Preprint MT-517 (1981).

[7] D. S. Lubinsky and A. Sidi, Convergence of linear and nonlinear Padé approximants from series of orthogonal polynomials, Technion Computer Science Preprint (1981).

[8] S. P. Suetin, On the convergence of rational approximations to polynomial expansions in domains of meromorphy of a given function, Math. USSR. Sb., 34 (1978), 367-381.

[9] H. Wallin, Potential theory and approximation of analytic functions by rational interpolation, Proceedings of the Colloquium on Complex Analysis at Joensuu. Lecture Notes in Mathematics, 747 (1979), 434-450.

[10] The convergence of Pade approximants and the size of the power series coefficients, Applicable Analysis, 4 (1974), 235-251.

[11] J. Walsh, The location of critical points of analytic and harmonic functions, Amer. Math. Soc., 34, New York, 1950.

Received December 10, 1981. Research supported by Lady Davis Fellowship.

TECHNION-IIT

32000 HAIFA, ISRAEL 



\section{PACIFIC JOURNAL OF MATHEMATICS EDITORS}

Donald BABBITT (Managing Editor)

University of California

Los Angeles, CA 90024

Hugo Rossi

University of Utah

Salt Lake City, UT 84112

C. C. Moore and Arthur Ogus

University of California

Berkeley, CA 94720
J. DugundiI

Department of Mathematics

University of Southern California

Los Angeles, CA 90089-1113

R. Finn and H. SAMELSON

Stanford University

Stanford, CA 94305

ASSOCIATE EDITORS
R. ARENS
E. F. BECKENBACH
B. H. NeUmanN
F. WOLF
K. YosHIDA (1906-1982)

\section{SUPPORTING INSTITUTIONS}

UNIVERSITY OF ARIZONA

UNIVERSITY OF BRITISH COLUMBIA

CALIFORNIA INSTITUTE OF TECHNOLOGY

UNIVERSITY OF CALIFORNIA

MONTANA STATE UNIVERSITY

UNIVERSITY OF NEVADA, RENO

NEW MEXICO STATE UNIVERSITY

OREGON STATE UNIVERSITY
UNIVERSITY OF OREGON

UNIVERSITY OF SOUTHERN CALIFORNIA

STANFORD UNIVERSITY

UNIVERSITY OF HAWAII

UNIVERSITY OF TOKYO

UNIVERSITY OF UTAH

WASHINGTON STATE UNIVERSITY

UNIVERSITY OF WASHINGTON 


\section{Pacific Journal of Mathematics}

\section{Vol. 108, No. $1 \quad$ March, 1983}

Waleed A. Al-Salam and A. Verma, $q$-Konhauser polynomials $\ldots \ldots \ldots \ldots 1$

Alfred David Andrew, The Banach space JT is primary $\ldots \ldots \ldots \ldots \ldots . . .6$

Thomas E. Bengtson, Bessel functions on $P_{n} \ldots \ldots \ldots \ldots \ldots \ldots$

Joaquim Bruna Floris and Francesc Tugores, Free interpolation for

holomorphic functions regular to the boundary $\ldots \ldots \ldots \ldots \ldots \ldots \ldots \ldots$

Peter Dierolf and Susanne Dierolf, Topological properties of the dual pair

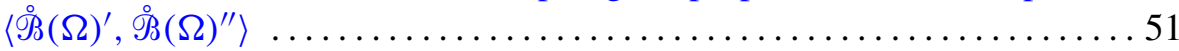

Gerald Arthur Edgar, An ordering for the Banach spaces $\ldots \ldots \ldots \ldots \ldots 83$

Basil Gordon, A proof of the Bender-Knuth conjecture . . . . . . . . . . . . . 99

Harold T. Hodes, A minimal upper bound on a sequence of Turing degrees

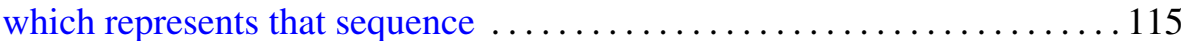

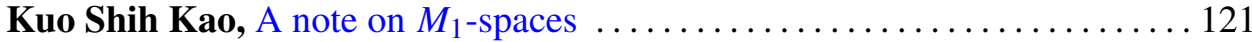

Frank Kost, Topological extensions of product spaces ................ 129

Eva Lowen-Colebunders, On the convergence of closed and compact

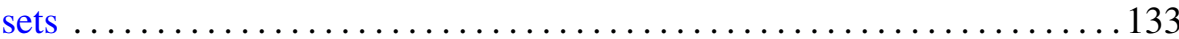

Doron Lubinsky, Divergence of complex rational approximations . . . . . . 141

Warren May and Elias Hanna Toubassi, Endomorphisms of rank one

mixed modules over discrete valuation rings $\ldots \ldots \ldots \ldots \ldots \ldots \ldots \ldots \ldots$

Richard Patrick Morton, The quadratic number fields with cyclic

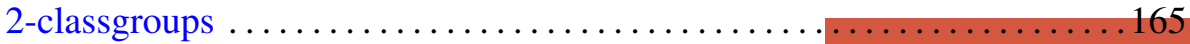

Roderic Murufas, Rank of positive matrix measures . . . . . . . . . . . . 177

Helga Schirmer, Fixed point sets of homotopies . . . . . . . . . . . . . 191

E. Taflin, Analytic linearization of the Korteweg-de Vries equation ........ 203

James Thomas Vance, Jr., $L^{p}$-boundedness of the multiple Hilbert

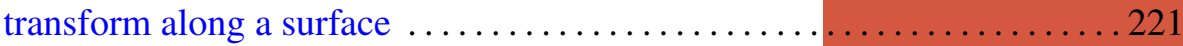

Hiroshi Yamaguchi, A property of some Fourier-Stieltjes transforms . . . . . 243 